ARTICLE

Received 30 Jun 2015 | Accepted 12 Aug 2015 | Published 18 Sep $2015 \quad$ DOl: 10.1038/ncomms9348 OPEN

\title{
Coordination templated $[2+2+2]$ cyclotrimerization in a porous coordination framework
}

Yong-Sheng Wei ${ }^{1}$, Mei Zhang ${ }^{1}$, Pei-Qin Liao ${ }^{1}$, Rui-Biao Lin' ${ }^{1}$, Tai-Yang Li', Guang Shao', Jie-Peng Zhang ${ }^{1}$ \& Xiao-Ming Chen ${ }^{1}$

Controlling chemical reactions by the supramolecular confinement effects of nanopores has attracted great attention. Here we show that open metal sites in porous coordination frameworks can constitute more powerful and strict templates for precision syntheses. A Fe(III) dicarboxylate framework functionalized with triangularly arranged metal sites is used to accomplish [2+2+2] cyclotrimerization reactions for organonitrile, alkyne and alkene monomers bearing a geometrically suitable pyridyl group. In situ single-crystal X-ray diffraction facilitates the direct observation of such a coordination templated reaction, before cylcotrimerization, the monomer coordinates at the Fe(III) centre by its pyridyl donor, which forces three unsaturated groups to gather around a position very similar with that of the desired covalent cyclic trimer. After the reaction, the trimers serve as tripodal ligands to perfectly fix the $\mathrm{Fe}(\mathrm{III})$ ions and the whole crystal to generate an exceptionally rigid and porous material with large surface area coupled with guest-proof zero thermal expansion.

\footnotetext{
${ }^{1}$ MOE Key Laboratory of Bioinorganic and Synthetic Chemistry, School of Chemistry \& Chemical Engineering, Sun Yat-Sen University, Guangzhou 510275, China. Correspondence and requests for materials should be addressed to J.-P.Z. (email: zhangjp7@mail.sysu.edu.cn).
} 
B esides many useful applications such as storage, separation, catalysis, and so on, porous materials may also serve as nano-reactors to control the molecular structures and morphologies of the products by restricting the space around the guest reactants/products, and even form host-guest nanocomposites with interesting properties ${ }^{1-3}$. Compared with the conventional bulk reactions, the structrues, molecular weights and stereoregularity of polymer products obtained in porous hosts ${ }^{4}$, especially periodic/crystalline ones with microporous channels ${ }^{5-7}$, can be regulated to much higher levels. When the reactant and products are both small molecules, organic reactions such as Diels-Alder addition ${ }^{8}$, isomerization ${ }^{9}$ and photodimerization $^{10}$ can be more rationally controlled ${ }^{11}$, because the guest molecules can fit the periodicity of the pore environment to enable good host-guest matching ${ }^{12}$. More importantly, some reaction stages may be directly visualized by single-crystal X-ray diffraction (SCXRD) ${ }^{11,13,14}$, although keeping the sample single-crystallinity after physical/chemical changes is always a great challenge ${ }^{15-23}$.

Because the pore and the guest recognize each other by hydrogen bonding, $\pi-\pi$ stacking, and more commonly other weaker supramolecular interactions, their sizes and shapes must be commensurate so that the guest reactants can be fixed at the right positions to achieve good control over the chemical reaction. In other words, the host always loses porosity after carrying out such precision chemical syntheses. On the other hand, by virtue of the strong and highly directional coordination bonds, metal ions and organic bridging ligands can self-assemble to build crystalline open frameworks (well known as porous coordination polymers or metal-organic frameworks) with overwhelmingly high porosity and robustness compared with other molecular materials ${ }^{24-29}$. In many cases, the coordination sites of the metal ions are not fully used by the bridging ligands. The unoccupied or open metal sites can bind guest molecules strongly at specific positions/directions, which should be ideal for development of strictly controlled reactors/reactions. Herein we prove this concept by using a prototypical material $\left[\mathrm{Fe}_{3}\left(\mu_{3}-\mathrm{O}\right)(\mathrm{bdc})_{3} \mathrm{X}\left(\mathrm{H}_{2} \mathrm{O}\right)_{2}\right]$ (MIL-88B (refs 30-32); $\mathrm{H}_{2}$ bdc $=1$,4-benzenedicarboxylic acid, $\left.\mathrm{X}=\mathrm{OH}^{-} / \mathrm{Cl}^{-} / \mathrm{F}^{-}\right)$as the host coordination template. A series of facile and strictly selective $[2+2+2]$ coordination templated cyclotrimerization (CTC) reactions have been realized. Unlike other molecular reactors, the reaction can drastically improve the gas adsorption performance of the host crystal.

\section{Results}

Synthesis and structure. MIL-88B is well known for its highly flexible behaviours towards various organic molecules ${ }^{30-32}$. An interesting structural feature of MIL-88B is that every three adjacent $\mathrm{Fe}$ (III) ions locating at the same channel (from three $\mathrm{Fe}_{3}\left(\mu_{3}-\mathrm{O}\right)$ clusters) defines a regular triangle with converging $\mathrm{Fe}-$ $\mathrm{H}_{2} \mathrm{O} / \mathrm{X}$ vectors. The highly directional coordination bonds of the octahedral $\mathrm{Fe}$ (III) ions with such an alignment may fix three molecules in an ideal configuration to facilitate the $[2+2+2]$ CTC reactions (Fig. 1). Considering the geometry of such a $\mathrm{Fe}_{3}$ coordination template, we selected 4-cyanopyridine (4-pyCN), 4-ethynylpyridine $\left(4-\mathrm{pyC}_{2} \mathrm{H}\right)$ and 4-vinylpyridine (4-pyC $\mathrm{H}_{3}$ ), each bearing a coordination donor at one end and an unsaturated group at the opposite end, as the reactants or monomers.

The CTC reactions were accomplished by loading the monomers in microcrystalline MIL-88B, removing excess monomers outside the microcrystal host, and then heating at $140^{\circ} \mathrm{C}$ within $12 \mathrm{~h}$, or simply heating a mixture of MIL-88B and excess monomers at the same condition. Powder X-ray diffraction (PXRD) showed that, the unit-cell volumes of the resultant crystalline products are even larger than that of the most-open form of MIL-88B (Supplementary Figs 2 and 3) ${ }^{31,32}$. The desired trisubstituted $s$-triazine, benzene, and cyclohexane derivatives, namely 2,4,6-tri(pyridin-4-yl)-1,3,5-triazine (tpt), 1,3,5-tri(pyridin4-yl)benzene (tpb) and 1,3,5-tri(pyridin-4-yl)cyclohexane (tpc), were first detected by infrared (IR) spectroscopy and electrospray ionization mass spectrometry (ESI-MS) analyses of the resultant crystalline products (Supplementary Figs 4 and 5). The formation of these cyclic trimers inside the $\mathrm{Fe}_{3}$ coordination templates was further confirmed by SCXRD. It should be noted that, MIL-88B has been always synthesized as microcrystalline powders ${ }^{30,33}$, but we now succeeded to grow large single crystals by using hydrofluoric acid to replace the conventionally used $\mathrm{NaOH}$ reactant. Finally, the organic products were recovered from the acid-digested crystalline products and fully characterized by IR, ESI-MS, ${ }^{1} \mathrm{H}$ and ${ }^{13} \mathrm{C}$ nuclear magnetic resonance (NMR) spectroscopy (Supplementary Figs 6-11).

On the basis of the SCXRD data, the chemical formulae of the product crystals obtained by the CTC reactions are determined as $\left[\mathrm{Fe}_{3}\left(\mu_{3}-\mathrm{O}\right)(\mathrm{bdc})_{3}(\mathrm{tpt})\right] \mathrm{X}(\mathrm{MIL}-88 \mathrm{~B}-\mathrm{tpt}),\left[\mathrm{Fe}_{3}\left(\mu_{3}-\mathrm{O}\right)(\mathrm{bdc})_{3}(\mathrm{tpb})\right] \mathrm{X}$ (MIL-88B-tpb) and $\left[\mathrm{Fe}_{3}\left(\mu_{3}-\mathrm{O}\right)(\mathrm{bdc})_{3}(\mathrm{tpc})\right] \mathrm{X}$ (MIL-88B-tpc), respectively, (Fig. 2 and Supplementary Table 1). Besides the original 6-connected acs networks of $\left[\mathrm{Fe}_{3}\left(\mu_{3}-\mathrm{O}\right)(\mathrm{bdc})_{3}\right]$, each $\mathrm{Fe}_{3}$ coordination template embeds a tpt/tpb/tpc ligand, which coordinates with the three Fe(III) ions by its three pyridyl ends (Fig. 2 and Supplementary Fig. 1). The coordination networks of the product crystals possess a rare binodal $(3,9)$-connected nia-d topology ${ }^{34}$, regarding the tpt/tpb/tpc ligands and the $\mathrm{Fe}_{3}$ clusters as 3- and 9-connected nodes, respectively. Interestingly, the unitcell volumes of the product crystals are ca. 145, 18 and 9\% larger than those of the dry, as-synthesized, and most-open forms of MIL-88B, respectively ${ }^{31}$, because the large tpt/tpb/tpc ligands force the host framework to expand to an extent not possible for lattice guest molecules.

Reaction mechanism. To verify the CTC reaction mechanism, control experiments were performed. Using 3-cyanopyridine (3-pyCN) and benzonitrile (PhCN) as monomers (Fig. 3d,e), the trimeric product of 3-pyCN/PhCN was not detected by either IR or ESI-MS of the reaction mixtures (Supplementary Figs 12 and 13). When a mixture of 4-pyCN and PhCN was used to perform the same reaction, only tpt was detected among four possible trimeric products (Fig. 3f and Supplementary Fig. 14). These results confirmed that MIL-88B is not able to catalyse the trimerization reactions involving monomers with unsuitable coordination sites and molecular geometry. More importantly, when excess 4-pyCN monomers were used for the CTC reaction, tpt cannot be detected out of the $\mathrm{Fe}_{3}$ coordination templates (Supplementary Fig. 15), meaning that in MIL-88B-tpt, the trimeric product tpt is solely generated at the $\mathrm{Fe}_{3}$ coordination templates. Note that the CTC reactions can be furnished in very high yields $(>90 \%)$ without using excess monomers (Supplementary Figs 3-5), confirming the rule of the $\mathrm{Fe}_{3}$ coordination template and exclude the possibilities of cyclotrimerization at other sites such as a single metal centre locating at defects and/or outer surface of the crystals.

To reveal more details for the CTC reaction mechanism, we carried out an in situ SCXRD study. The unit-cell volume of the crystal stabilized at a value of $16 \%$ larger than that of assynthesized MIL-88B but $1.0 \%$ smaller than MIL-88B-tpt, after heated to $110^{\circ} \mathrm{C}$ for $24 \mathrm{~h}$. Structure analysis revealed a stable composition of $\left[\mathrm{Fe}_{3}\left(\mu_{3}-\mathrm{O}\right)(\mathrm{bdc})_{3}(4-\mathrm{pyCN}) \mathrm{X}\left(\mathrm{H}_{2} \mathrm{O}\right)\right]$ (denoted as 4-pyCN@MIL-88B, Supplementary Table 1), indicating that one third of terminal $\mathrm{H}_{2} \mathrm{O} / \mathrm{X}$ ligands are substituted by 4 -pyCN molecules. From the crystal structure (Fig. 2b), it can be clearly seen that 4-pyCN coordinates at the Fe(III) ion using its pyridyl 


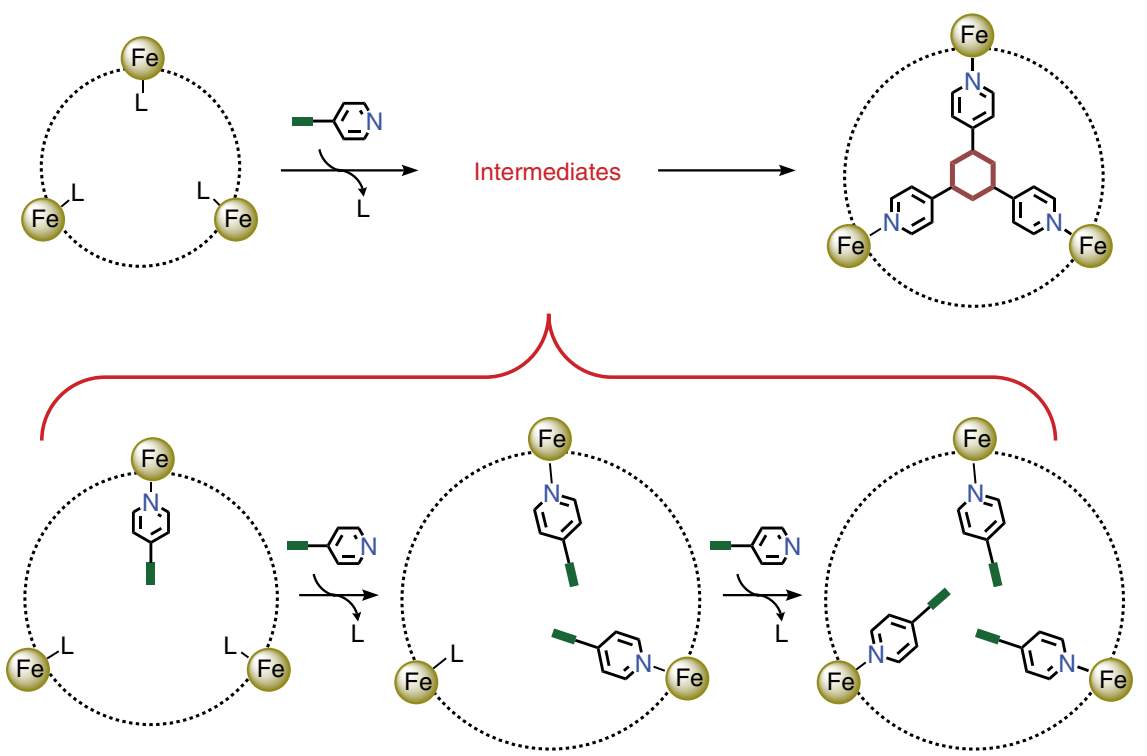

Figure 1 | The proposed CTC reaction mechanism. The monomer molecule bearing an unsaturated functional group (thick green stick) and an anchor (the pyridyl group) replaces the terminal ligand (L) originally coordinated at the Fe(III) site. The convergent and triangular arrangement of the Fe(III) sites forces every three unsaturated groups of the monomer ligands to gather in a close configuration suitable for the cyclotrimerization reaction. The ligand replacement occurs at a step-by-step manner.

a

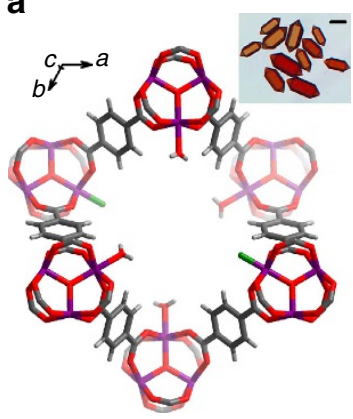

d

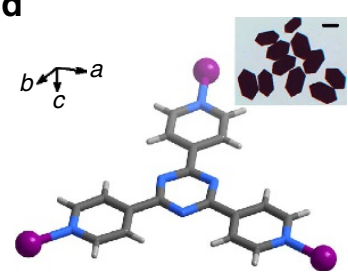

b

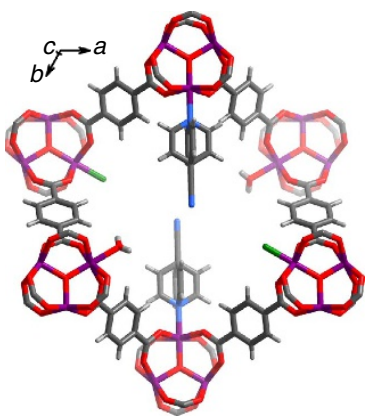

e

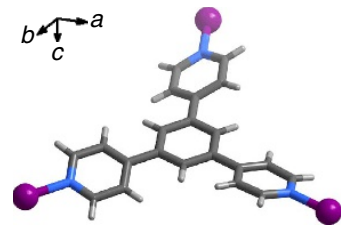

C

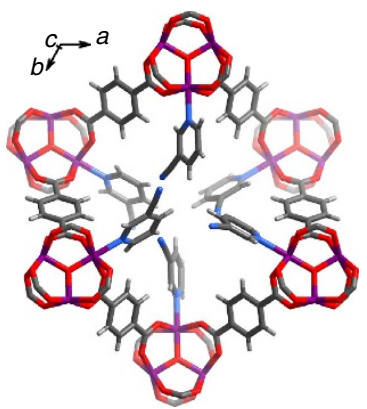

f

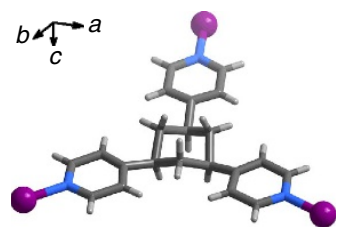

Figure 2 | X-ray single-crystal structures. (a) MIL-88B. (b) 4-pyCN@MIL-88B. (c) 3-pyCN@MIL-88B. (d-f) The trimers tpt, tpb and tpc embedded in MIL-88B-tpt, MIL-88B-tpb and MIL-88B-tpc, respectively. Color codes: dark grey, C; light grey, H; blue, N; red, O; purple, Fe; green, F. The pyridyl ring of 4-pyCN is two-fold disordered at conformations parallel and perpendicular with the $\mathrm{Fe}_{3}$ plane. Some other crystal structures are symmetrically disordered, only one possible structure is shown for clarity. Inset: photographs of corresponding compounds (Scale bars, $0.10 \mathrm{~mm}$ ).

$\mathrm{N}$ donor, so that its cyano group is pointed at the centre of the $\mathrm{Fe}_{3}$ coordination template. The pyridyl ring of $4-\mathrm{pyCN}$ in 4 pyCN@MIL-88B showed obvious conformational disorder, because the pyridyl $\mathrm{C}-\mathrm{H}$ moieties has no specific interaction with other parts. In contrast, the pyridyl and triazine rings of tpt in MIL-88B-tpt are strictly coplanar (Fig. 2d). Further increasing the heating time and/or temperature resulted in monotonic and small increase of the unit-cell volume until a stable value virtually identical with that of MIL-88B-tpt, which was not changed after heated at $180^{\circ} \mathrm{C}$ for $24 \mathrm{~h}$. As shown in Supplementary Fig. 16, similar phenomena can be also observed by PXRD. Structure analysis confirmed that the crystal at this stage is identical with
MIL-88B-tpt (Supplementary Table 1), meaning that the cyclotrimerization reaction was completed.

For comparison, 3-pyCN was also used for the in situ SCXRD study. Structure analysis of the crystal above the melting point of 3 -pyCN revealed a chemical formula of $\left[\mathrm{Fe}_{3}\left(\mu_{3}-\mathrm{O}\right)(\mathrm{bdc})_{3}\right.$ (3-pyCN) $)_{3}$ X (denoted as 3-pyCN@MIL-88B, Supplementary Table 1), meaning that each Fe(III) ion is coordinated by one 3-pyCN, or all terminal $\mathrm{H}_{2} \mathrm{O} / \mathrm{X}$ ligands are completely substituted. Because the cyano group locates at the meta-position of the pyridyl ring, there is no steric hindrance among three 3-pyCN ligands in the same $\mathrm{Fe}_{3}$ coordination template (Fig. 2c). Unlike 4pyCN, the pyridyl ring of 3 -pyCN is almost perpendicular with 
a
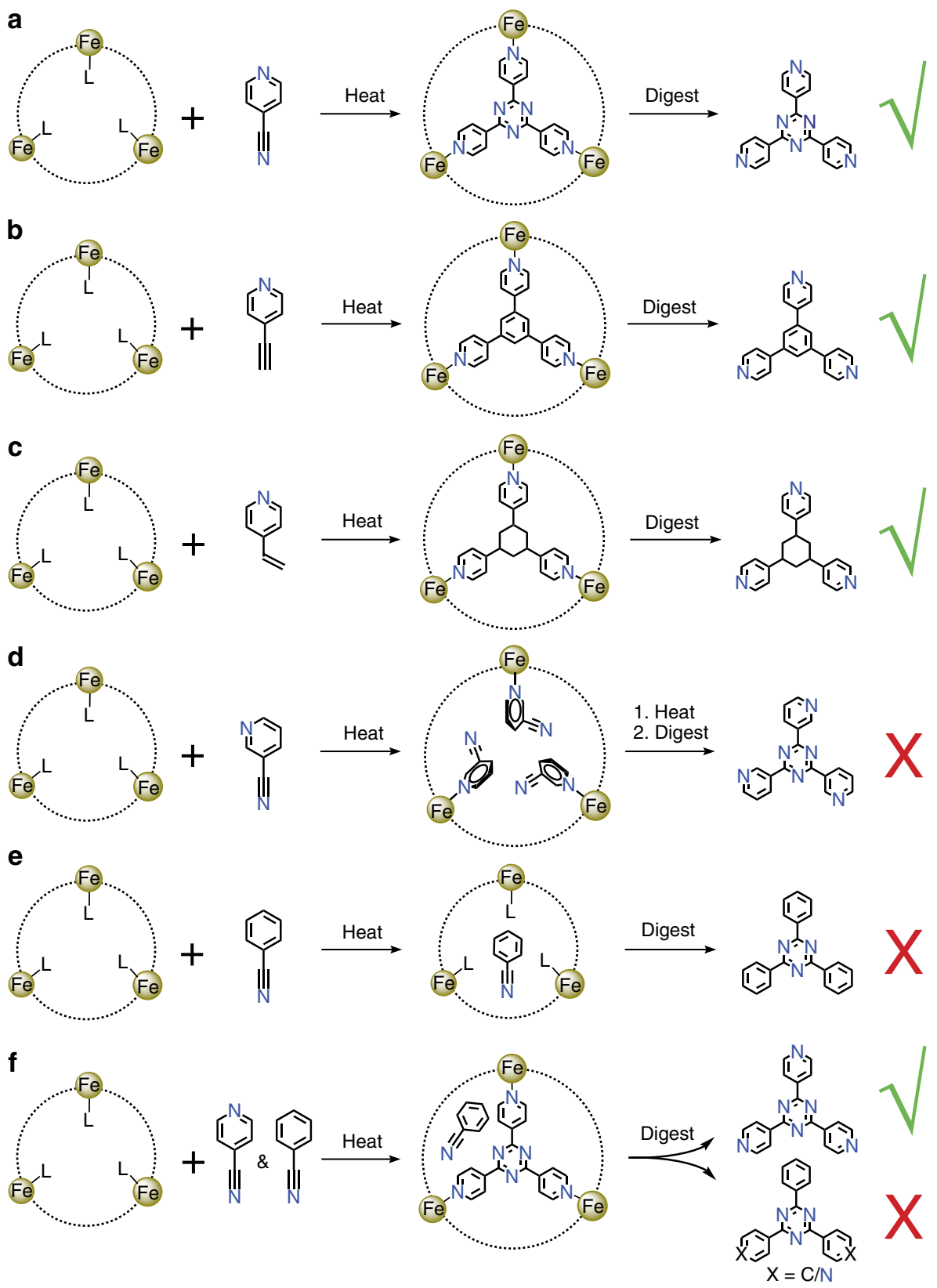

Figure 3 | Summary of the CTC reactions for various monomers. (a-c) When 4-pyCN, 4-py $\mathrm{C}_{2} \mathrm{H}$, and 4-py $\mathrm{C}_{2} \mathrm{H}_{3}$ were used as monomers, the corresponding trimeric products were observed by ESI-MS, IR, ${ }^{1} \mathrm{H} N M R,{ }^{13} \mathrm{C} N M R$ and SCXRD. (d) When 3-pyCN was used as the monomer, the coordination of 3-pyCN molecules on the Fe(III) centre was observed by SCXRD, but the expected trimeric product could not be detected by ESI-MS, IR or SCXRD. (e) When PhCN was used as the monomer, the expected trimeric product was not detected by ESI-MS, IR or SCXRD. (f) When a mixture of 4-pyCN and PhCN was used as monomers, only tpt was detected by ESI-MS among four possible trimeric products.

the $\mathrm{Fe}_{3}$ plane, because with this conformation the cyano group can form weak hydrogen bonds $(\mathrm{N} \cdots \mathrm{C} 3.81(4) \AA)$ with the pyridyl ring of a neighbour locating at another $\mathrm{Fe}_{3}$ coordination template (Supplementary Fig. 17). In other words, the cyano groups of 3-pyCN monomers are evenly distributed in 3pyCN@MIL-88B and fixed at positions distinctly unfit for the trimerization reaction. Consequently, the corresponding triazine product of 3-pyCN cannot be obtained by further increasing the heating time/temperature.

Modification of flexibility and porosity. Due to the additional tripodal ligands, the product crystals obtained by the CTC reactions display very different framework flexibility and adsorption property compared with MIL-88B. Thermogravimetry analyses and variable-temperature PXRD showed that MIL-88B-tpt is a rigid framework stable up to $400{ }^{\circ} \mathrm{C}$ (Supplementary Figs 18 and 19). Variable-temperature SCXRD at $100-455 \mathrm{~K}$ showed that guest and temperature can barely affect the unit-cell parameters of MIL-88B-tpt, giving zero thermal expansion across the $a b$ plane and very small negative thermal expansion along the $c$-axis (Fig. 4a and Supplementary Figs $19 \mathrm{~b}$ and 20), which is exceptional for such a porous structure ${ }^{35-37}$. Further, guest-free MIL-88B-tpt showed a type-I $\mathrm{N}_{2}$ isotherm, corresponding with a Brunauer-Emmett-Teller surface area of $1,113 \mathrm{~m}^{2} \mathrm{~g}^{-1}$ and a Langmuir surface area of $1,331 \mathrm{~m}^{2} \mathrm{~g}^{-1}$, as well as a pore volume of $0.56 \mathrm{~cm}^{3} \mathrm{~g}^{-1}$. In contrast, guest-free MIL-88B showed a type-II $\mathrm{N}_{2}$ isotherm with very small uptake, surface area, and pore 


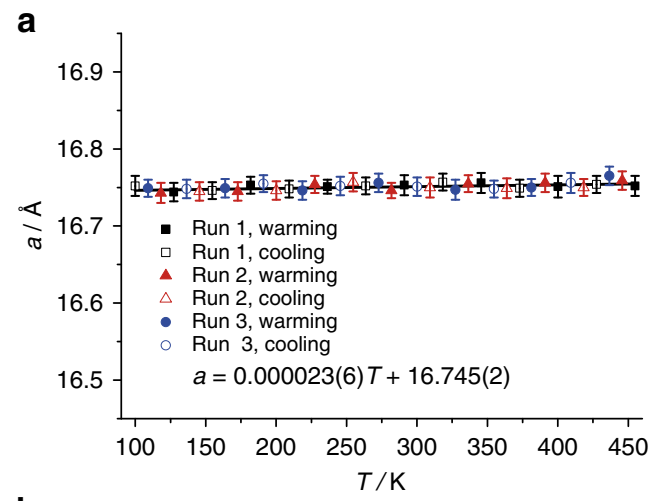

b

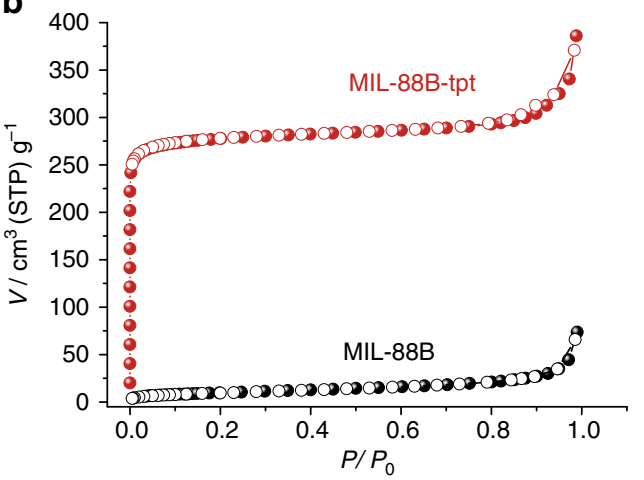

Figure 4 | Framework flexibility and gas adsorption property of the hostguest composite. (a) Temperature- and guest-dependences of the $a$-axis length of MIL-88B-tpt obtained in several heating/cooling cycles. The as-synthesized crystal of MIL-88B-tpt was used to start the measurement. Obviously, the $a$-axis length is nearly unaffected by guest and temperature, giving zero thermal expansion across the $a b$-plane. (b) $\mathrm{N}_{2}$ adsorption (filled) and desorption (open) isotherm of MIL-88B and MIL-88B-tpt.

volume (Fig. 4b), because the shrunk coordination framework of MIL-88B can be hardly opened by $\mathrm{N}_{2}$ molecules ${ }^{38}$, although it can undergo significant solvent-induced breathing.

\section{Discussion}

In conventional approaches, cyano, alkyne and alkene groups are quite different in reactivity. Cyclotrimerization of organonitriles to form 1,3,5-triazine derivatives is stereo selective, but both catalyst and high reaction temperature $\left(>200^{\circ} \mathrm{C}\right)$ are required ${ }^{39,40}$. The synthesis of benzene derivatives through catalysed cyclotrimerization of alkynes, which usually forms 1,2,4- and 1,3,5-trisubstituted isomers as a mixture, concomitant with polymers as byproducts, has been studied extensively ${ }^{41}$. Without catalyst, alkenes can easily polymerize to form long chains. On the other hand, when two alkene molecules are properly aligned (usually in a crystal) with separation less than $4.1 \AA$, photo/thermochemical [2+2] cycloaddition can occur to generate cyclobutane with high stereo selectivity ${ }^{9,15}$. Nevertheless, by using weak supramolecular interactions and pore confinement effects, three alkene molecules can be hardly fixed in a configuration suitable for $[2+2+2]$ cyclotrimerization, so that cyclohexanes are generally obtained by hydrogenation of benzenes. Obviously, MIL-88B greatly facilitates $[2+2+2]$ cyclotrimerization reactions of organonitrile, alkyne, and alkene monomers bearing a geometrically suitable pyridyl group, highlighting the coordination templating effects of its open metal sites.

The results of in situ SCXRD showed that the intermediate state with a formula of $\left[\mathrm{Fe}_{3}\left(\mu_{3}-\mathrm{O}\right)(\mathrm{bdc})_{3}(4-\mathrm{pyCN})_{3}\right] \mathrm{X}$, that is, three 4-pyCN monomers simultaneously coordinate at the same $\mathrm{Fe}_{3}$ coordination template, cannot be observed by diffraction techniques that are suitable for characterizing thermodynamic equilibrium structures ${ }^{15-19}$. Obviously, the supramolecular trimer $(4-\mathrm{pyCN})_{3}$ has a larger size compared with the covalent trimer tpt, meaning that the unit-cell volume of $\left[\mathrm{Fe}_{3}\left(\mu_{3}-\mathrm{O}\right)(\mathrm{bdc})_{3}\right.$ $\left.(4-\mathrm{pyCN})_{3}\right] \mathrm{X}$ needs to be larger than that of MIL-88B-tpt. However, the unit-cell volume of the most-open form of MIL-88B, obtained by inclusion of large organic molecules ${ }^{31,32}$, is still smaller than that of MIL-88B-tpt, indicating that $\left[\mathrm{Fe}_{3}\left(\mu_{3}-\mathrm{O}\right)(\mathrm{bdc})_{3}(4-\mathrm{pyCN})_{3}\right] \mathrm{X}$ with an even larger unit-cell volume should be energetically unfavourable, at least at the thermodynamic equilibrium state. In other words, such a theoretical intermediate state may be too reactive, which occurs transiently and transforms to the trimeric product immediately. Therefore, the CTC reaction might be mainly controlled by the ligand substitution reaction rather than the organic reaction. Indeed, it has been demonstrated that other organic molecules, including pyridine (with a stronger coordination ability compared with 4-pyCN), always serve as pure lattice guests, and cannot substitute the $\mathrm{H}_{2} \mathrm{O} / \mathrm{X}$ ligand in all MIL-88 series at room temperature ${ }^{31,32}$. On the other hand, while there is no conventional catalytic site in MIL-88B, the 4-pyCN to tpt transformation occurs at a relatively low temperature, which implies that the flexible coordination network and the $\mathrm{Fe}_{3}$ coordination template, having a large tendency to shrink at the transient intermediate state $\left[\mathrm{Fe}_{3}\left(\mu_{3}-\mathrm{O}\right)(\mathrm{bdc})_{3}(4-\mathrm{pyCN})_{3}\right] \mathrm{X}$, might compress the supramolecular trimer (4-pyCN) $)_{3}$ and force it to form the covalent trimer tpt.

In summary, we demonstrated that with a judicious geometric consideration of the host-guest structure, the open metal sites in a porous coordination framework can be rationally used to fix guest molecules to facilitate chemical reaction difficult or even impossible for conventional methods and other nano-reactors. Importantly, the template crystal can retain single-crystallinity throughout the supramolecular and molecular reaction processes, allowing the direct observation of several key reaction stages. Also interestingly, the precisely controlled reaction can drastically improve the framework rigidity and gas adsorption performance of the crystalline host. These results should be instructive for further exploration of new chemical reactions and functions using porous crystals as a platform.

\section{Methods}

Materials and physical measurements. Reagents were commercially available and used without further purification except otherwise stated. The monomer 4-pyC ${ }_{2} \mathrm{H}_{3}$ was distilled to remove polymerization inhibitor hydroquinone before use. ESI-MS was obtained using a Shimadzu LCMS-2010A mass spectrometer, for which samples were digested by sonication in a mixture of hydrochloric acid and $\mathrm{MeOH}$ after removal of the residual monomers by sublimation under vacuum. Elemental analyses (EA) were conducted using an Elementar Vario EL analyzer using guest-free samples (with a little water adsorbed from air). IR spectra were recorded on a Bruker TENSOR 27 FT-IR spectrometer in the $400-4,000 \mathrm{~cm}^{-1}$ region with $\mathrm{KBr}$ pellets. ${ }^{1} \mathrm{H} \mathrm{NMR}$ and ${ }^{13} \mathrm{C}$ NMR spectra were recorded on a Bruker AVANCE III $400 \mathrm{MHz}$ NMR spectrometer. Chemical shifts were quoted in p.p.m. referenced to the appropriate solvent peak or 0.0 p.p.m. for tetramethylsilicane. PXRD patterns were collected on a Bruker D8-Advance diffractometer with $\mathrm{Cu} \mathrm{K} \alpha$ radiation and a LynxEye detector. Thermogravimetry analyses were performed on a TA Q50 system under $\mathrm{N}_{2}$ at a heating rate of $5{ }^{\circ} \mathrm{C} \min . \mathrm{N}_{2}$ sorption isotherms were measured by a Micromeritics ASAP 2020M physisorption analyzer.

Synthesis of MIL-88B. Microcrystals ${ }^{30,33}$ : A mixture of $\mathrm{FeCl}_{3} \cdot 6 \mathrm{H}_{2} \mathrm{O}(4.32 \mathrm{~g}$, $16.0 \mathrm{mmol})$ and $\mathrm{H}_{2} \mathrm{bdc}(2.66 \mathrm{~g}, 16.0 \mathrm{mmol})$ were dispersed in $\mathrm{N}, \mathrm{N}$-dimethylformamide (DMF; $80.0 \mathrm{ml}$ ) with $2 \mathrm{M} \mathrm{NaOH}(6.4 \mathrm{ml})$. The mixture was placed in a $100-\mathrm{ml}$ vial and sealed with a screw cap, which was heated at $100^{\circ} \mathrm{C}$ for $12 \mathrm{~h}$. Then, the orange solid was filtered, washed with DMF, and dried in air overnight to give $\left[\mathrm{Fe}_{3}\left(\mu_{3}-\mathrm{O}\right) \mathrm{Cl}\left(\mathrm{H}_{2} \mathrm{O}\right)_{2}(\mathrm{bdc})_{3}\right] \cdot$ guest (yield $\left.60 \%\right)$. The content of chloride ion was determined by potentiometric titration with the acid-digested samples. Large single crystals: a mixture of $\mathrm{FeCl}_{3} \cdot 6 \mathrm{H}_{2} \mathrm{O}(0.081 \mathrm{~g}, 0.3 \mathrm{mmol}), \mathrm{H}_{2} \mathrm{bdc}(0.050 \mathrm{~g}, 0.3 \mathrm{mmol})$, 
hydrofluoric acid ( $0.1 \mathrm{ml}, 49 \mathrm{wt} \%$ in $\left.\mathrm{H}_{2} \mathrm{O}\right)$, and DMF $(3.0 \mathrm{ml})$ was stirred for $30 \mathrm{~min}$ in air, then transferred and sealed in a 20 -ml vial with a screw cap, which was heated at $90^{\circ} \mathrm{C}$ for 3 days and then cooled to room temperature, giving orange block crystals, $\left[\mathrm{Fe}_{3}\left(\mu_{3}-\mathrm{O}\right) \mathrm{F}_{0.74}(\mathrm{OH})_{0.26}\left(\mathrm{H}_{2} \mathrm{O}\right)_{2}(\mathrm{bdc})_{3}\right] \cdot$ guest (yield 50\%). The content of fluoride ion was determined by the fluoride selective electrode with the acid-digested samples.

Coordination templated cyclotrimerization reactions. The as-synthesized microcrystals of MIL-88B were exchanged with hot $\mathrm{MeOH}$ using a Soxhlet extractor to remove the unreacted $\mathrm{FeCl}_{3}$ and $\mathrm{H}_{2} \mathrm{bdc}$ in the channels. The reaction method without using excess monomers: the monomer was loaded into MIL-88B $(0.100 \mathrm{~g})$ by mixing it with 4 -pyCN $(1.00 \mathrm{~g})$ and heated at $110^{\circ} \mathrm{C}$ for $24 \mathrm{~h}$ immersing it in an anhydrous toluene solution $(20.0 \mathrm{~mL})$ of $4-\mathrm{pyC}_{2} \mathrm{H}(0.100 \mathrm{~g})$ and heated at $50^{\circ} \mathrm{C}$ for $6 \mathrm{~h}$ under $\mathrm{N}_{2}$, or by immersing it in $4-\mathrm{pyC}_{2} \mathrm{H}_{3}(1.0 \mathrm{ml})$ at room temperature for 3 days; After removal of the excess monomers by sublimation for 4-pyCN or washing with $\mathrm{CH}_{2} \mathrm{Cl}_{2}$ for 4- $\mathrm{pyC}_{2} \mathrm{H}$ and 4- $\mathrm{pyC}_{2} \mathrm{H}_{3}$, the monomer-loaded MIL-88B was transferred and sealed in a 1-ml glass tube, heated in an oven at $140{ }^{\circ} \mathrm{C}$ for $12 \mathrm{~h}$ and then cooled to room temperature, giving black products (yields: $92 \%, 94 \%$ and $91 \%$, respectively). The reaction method using excess monomers: a mixture of MIL-88B $(0.100 \mathrm{~g})$ and the monomer, that is, 4-pyCN $(3.00 \mathrm{~g})$ or DMF $(3.0 \mathrm{ml})$ solution of $4-\mathrm{pyC}_{2} \mathrm{H}(0.140 \mathrm{~g})$ or $4-\mathrm{pyC}_{2} \mathrm{H}_{3}(3.0 \mathrm{ml})$ sealed in a $25-\mathrm{ml}$ teflon-lined stainless container, and the products were finally washed by DMF and dried in air (yield $>90 \%)$. MIL-88B-tpt: EA calc. (\%) for $\left[\mathrm{Fe}_{3}\left(\mu_{3}-\mathrm{O}\right)(\mathrm{bdc})_{3}(\mathrm{tpt})\right]$ $\mathrm{Cl} \cdot 9 \mathrm{H}_{2} \mathrm{O}\left(\mathrm{C}_{42} \mathrm{H}_{42} \mathrm{ClFe}_{3} \mathrm{~N}_{6} \mathrm{O}_{22}\right)$ : C, 43.14; $\mathrm{H}, 3.62 ; \mathrm{N}, 7.19$; found: $\mathrm{C}, 44.09 ; \mathrm{H}, 3.92$; $\mathrm{N}, 7.03$. MIL-88B-tpb: EA calc. (\%) for $\left[\mathrm{Fe}_{3}\left(\mu_{3}-\mathrm{O}\right)(\mathrm{bdc})_{3}(\mathrm{tpb})\right] \mathrm{Cl} \cdot 14 \mathrm{H}_{2} \mathrm{O}$ $\left(\mathrm{C}_{45} \mathrm{H}_{55} \mathrm{ClFe}_{3} \mathrm{~N}_{3} \mathrm{O}_{27}\right): \mathrm{C}, 42.46 ; \mathrm{H}, 4.36$; N, 3.30; found: $\mathrm{C}, 42.41 ; \mathrm{H}, 4.51$; $\mathrm{N}, 3.30$. MIL-88B-tpc: EA calc. (\%) for $\left[\mathrm{Fe}_{3}\left(\mu_{3}-\mathrm{O}\right)(\mathrm{bdc})_{3}(\mathrm{tpc})\right] \mathrm{Cl} \cdot 7 \mathrm{H}_{2} \mathrm{O}$ $\left(\mathrm{C}_{45} \mathrm{H}_{47} \mathrm{ClFe}_{3} \mathrm{~N}_{3} \mathrm{O}_{20}\right): \mathrm{C}, 46.88 ; \mathrm{H}, 4.11 ; \mathrm{N}, 3.64$; found: $\mathrm{C}, 47.41 ; \mathrm{H}, 4.24 ; \mathrm{N}, 3.57$. For samples used for SCXRD studies, the as-synthesized single crystals of MIL-88B were directly used, and the reactions were carried out at $180^{\circ} \mathrm{C}$ for $24 \mathrm{~h}$.

Separation of covalent trimers. The host-guest hybrid crystals were firstly dissolved in concentrated hydrochloric acid with stirring for $20 \mathrm{~min}$. After removing the undissolved white solid $\left(\mathrm{H}_{2} \mathrm{bdc}\right)$ by filtration, $\mathrm{pH}$ of the solution was adjusted to $\sim 11$ with aqueous $\mathrm{NaOH}(1 \mathrm{M})$. After that, the aqueous solution was extracted with $\mathrm{CH}_{2} \mathrm{Cl}_{2}$ for three times. The combined organic phase was dried over anhydrous $\mathrm{Mg}_{2} \mathrm{SO}_{4}$ and concentrated to remove solvent under reduced pressure, finally giving white products.

Single-crystal X-ray diffraction analyses. Single-crystal X-ray diffraction data were collected on an Oxford Gemini-S Ultra CCD-detector or a Rigaku XtaLAB P300DS-detector diffractometer $(\mathrm{Cu} \mathrm{K} \alpha)$. The measurement temperatures were controlled by a dry nitrogen flow using a Rigaku Gas Flow GN2 apparatus. For the in situ SCXRD study, a single crystal of MIL-88B and excess amount of monomer were sealed in a glass capillary. Nevertheless, identical single-crystal structure can be also obtained without using excess monomer. The diffraction data were collected just above the melting point of the monomer to avoid the interference of diffraction of crystalline monomer. All structures were solved by the direct method and refined with the full-matrix least-squares technique on $F^{2}$ by the SHELXTL software package. The terminal coordinated molecules/anions, namely, $\mathrm{H}_{2} \mathrm{O}, \mathrm{OH}^{-}$ and $\mathrm{F}^{-}$in MIL-88B, $\mathrm{H}_{2} \mathrm{O}, \mathrm{OH}^{-}, \mathrm{F}^{-}$and 4-pyCN in 4-pyCN@MIL-88B, are statistically and symmetrically disordered at the three terminal sites (crystallography three-fold symmetry) of one $\mathrm{Fe}_{3}\left(\mu_{3}-\mathrm{O}\right)(\mathrm{bdc})_{3}\left(\mathrm{~L}^{\mathrm{T}}\right)_{3}\left(\mathrm{~L}^{\mathrm{T}}=\right.$ terminal ligand). Since the donor atoms occupy essentially the same positions and cannot be distinguished by X-ray diffraction, they were refined using the EADP and EXYZ constraints. In 4-pyCN@MIL-88B and MIL-88B-tpc, the pyridyl rings of 4-pyCN and tpc are statistically/dynamically two-fold disordered at conformations parallel and perpendicular with the $\mathrm{Fe}_{3}$ plane. In MIL-88B-tpb, the pyridyl rings of tpb are statistically/dynamically/symmetrically two-fold disordered with molecular planes about $18^{\circ}$ with the $\mathrm{Fe}_{3}$ plane. In 3-pyCN@MIL-88B, the coordinated 3-pyCN molecule is symmetrically four-fold disordered because the bent molecule is locates at a two-fold axis and a mirror plane. Moreover, in MIL-88B-tpc, the non-planar cyclohexane ring is in the e,e,e-conformation and symmetrically two-fold disordered at the crystallography mirror plane. The SHELXTL restraint instructions DFIX, SIMU, ISOR and FLAT were applied to these disordered groups to keep their geometries and atomic displacement parameters reasonable. All host-framework non-hydrogen atoms were refined anisotropically. Hydrogen atoms were placed geometrically. The PLATON SQUEEZE treatment was applied because all lattice guests are extremely disordered and cannot be modelled. Detailed structure determination parameters and crystallographic data are given in Supplementary Table 1.

\section{References}

1. Farina, M., Silvestro, G. D. \& Sozzani, P. in Comprehensive Supramolecular Chemistry. (ed. Reinhoudt, D.) 371 (Pergamon: Oxford, 1996).

2. Sivaguru, J. et al. Asymmetric photoreactions within zeolites: role of confinement and alkali metal ions. Acc. Chem. Res. 36, 509-521 (2003).
3. Tajima, K. \& Aida, T. Controlled polymerizations with constrained geometries. Chem. Commun. (Camb) 2399-2412 (2000).

4. Soler-Illia, G. J. A. A. \& Azzaroni, O. Multifunctional hybrids by combining ordered mesoporous materials and macromolecular building blocks. Chem. Soc Rev. 40, 1107-1150 (2011).

5. Distefano, G. et al. Highly ordered alignment of a vinyl polymer by host-guest cross-polymerization. Nat. Chem. 5, 335-341 (2013).

6. Uemura, T., Uchida, N., Higuchi, M. \& Kitagawa, S. Effects of unsaturated metal sites on radical vinyl polymerization in coordination nanochannels. Macromolecules 44, 2693-2697 (2011).

7. Uemura, T., Yanai, N. \& Kitagawa, S. Polymerization reactions in porous coordination polymers. Chem. Soc. Rev. 38, 1228-1236 (2009).

8. Ikemoto, K., Inokuma, Y. \& Fujita, M. Diels-Alder via molecular recognition in a crystalline molecular flask. J. Am. Chem. Soc. 133, 16806-16808 (2011).

9. Yang, S.-Y. et al. Crystallographic snapshots of the interplay between reactive guest and host molecules in a porous coordination polymer: stereochemical coupling and feedback mechanism of three photoactive centers triggered by $\mathrm{UV}$-induced isomerization, dimerization, and polymerization reactions. J. Am Chem. Soc. 136, 558-561 (2013).

10. Zheng, S.-L., Pham, O., Vande Velde, C. M. L., Gembicky, M. \& Coppens, P. Competitive isomerization and dimerization in co-crystals of $1,1,6,6$ tetraphenyl-2,4-hexadiyne-1,6-diol and sorbic acid: a new look at stereochemical requirements for $[2+2]$ dimerization. Chem. Commun. (Camb) 2538-2540 (2008).

11. Inokuma, Y., Kawano, M. \& Fujita, M. Crystalline molecular flasks. Nat. Chem. 3, 349-358 (2011).

12. MacGillivray, L. R. et al. Supramolecular control of reactivity in the solid state: from templates to ladderanes to metal-organic frameworks. Acc. Chem. Res. 41 280-291 (2008).

13. Bloch, W. M. et al. Capturing snapshots of post-synthetic metallation chemistry in metal-organic frameworks. Nat. Chem. 6, 906-912 (2014).

14. Ikemoto, K., Inokuma, Y., Rissanen, K. \& Fujita, M. X-ray snapshot observation of palladium-mediated aromatic bromination in a porous complex. J. Am. Chem. Soc. 136, 6892-6895 (2014).

15. Kole, G. K. \& Vittal, J. J. Solid-state reactivity and structural transformations involving coordination polymers. Chem. Soc. Rev. 42, 1755-1775 (2013).

16. Zhang, J.-P., Liao, P.-Q., Zhou, H.-L., Lin, R.-B. \& Chen, X.-M. Single-crystal $\mathrm{X}$-ray diffraction studies on structural transformations of porous coordination polymers. Chem. Soc. Rev. 43, 5789-5814 (2014).

17. Kawano, M. \& Fujita, M. Direct observation of crystalline-state guest exchange in coordination networks. Coord. Chem. Rev. 251, 2592-2605 (2007).

18. Suh, M. P. \& Cheon, Y. E. Recent advances in the dynamics of single crystal to single crystal transformations in metal-organic open frameworks. Aust. J. Chem. 59, 605-612 (2006).

19. Halder, G. J. \& Kepert, C. J. Single crystal to single crystal structural transformations in molecular framework materials. Aust. J. Chem. 59, 597-604 (2006).

20. Yang, S. et al. Selectivity and direct visualization of carbon dioxide and sulfur dioxide in a decorated porous host. Nat. Chem. 4, 887-894 (2012).

21. Liu, T.-F. et al. Stepwise synthesis of robust metal-organic frameworks via postsynthetic metathesis and oxidation of metal nodes in a single-crystal to single-crystal transformation. J. Am. Chem. Soc. 136, 7813-7816 (2014).

22. Xiao, B. et al. Chemically blockable transformation and ultraselective lowpressure gas adsorption in a non-porous metal organic framework. Nat. Chem 1, 289-294 (2009).

23. Martí Gastaldo, C. et al. Side-chain control of porosity closure in single- and multiple-peptide-based porous materials by cooperative folding. Nat. Chem. 6, 343-351 (2014).

24. Furukawa, H., Cordova, K. E., O’Keeffe, M. \& Yaghi, O. M. The chemistry and applications of metal-organic frameworks. Science 341, 1230444 (2013).

25. Batten, S. R. et al. Terminology of metal-organic frameworks and coordination polymers (IUPAC Recommendations 2013). Pure Appl. Chem. 85, 1715-1724 (2013).

26. Zhou, H.-C. \& Kitagawa, S. Metal-organic frameworks (MOFs). Chem. Soc. Rev. 43, 5415-5418 (2014).

27. Devic, T. \& Serre, C. High valence $3 p$ and transition metal based MOFs. Chem Soc. Rev. 43, 6097-6115 (2014).

28. Kubota, R., Tashiro, S., Shiro, M. \& Shionoya, M. In situ X-ray snapshot analysis of transient molecular adsorption in a crystalline channel. Nat. Chem. 6, 913-918 (2014).

29. Cohen, S. M. Modifying MOFs: new chemistry, new materials. Chem. Sci. 1, 32-36 (2010).

30. Surble, S., Serre, C., Mellot-Draznieks, C., Millange, F. \& Ferey, G. A new isoreticular class of metal-organic-frameworks with the MIL-88 topology. Chem. Commun. (Camb) 284-286 (2006).

31. Serre, C. et al. Role of solvent-host interactions that lead to very large swelling of hybrid frameworks. Science 315, 1828-1831 (2007). 
32. Horcajada, P. et al. how linker's modification controls swelling properties of highly flexible iron(III) dicarboxylates MIL-88. J. Am. Chem. Soc. 133, 17839-17847 (2011).

33. McKinlay, A. C. et al. Nitric oxide adsorption and delivery in flexible MIL-88(Fe) metal-organic frameworks. Chem. Mater. 25, 1592-1599 (2013).

34. Zheng, S.-T. et al. Entrapment of metal clusters in metal-organic framework channels by extended hooks anchored at open metal sites. J. Am. Chem. Soc. 135, 10270-10273 (2013).

35. Zhou, H.-L. et al. Direct visualization of a guest-triggered crystal deformation based on a flexible ultramicroporous framework. Nat. Commun. 4, 2534 (2013).

36. Phillips, A. E., Goodwin, A. L., Halder, G. J., Southon, P. D. \& Kepert, C. J. Nanoporosity and exceptional negative thermal expansion in single-network cadmium cyanide. Angew. Chem. Int. Ed. 47, 1396-1399 (2008).

37. Goodwin, A. L., Chapman, K. W. \& Kepert, C. J. Guest-dependent negative thermal expansion in nanoporous prussian blue analogues

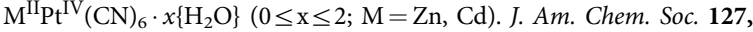
17980-17981 (2005).

38. Vuong, G.-T., Pham, M.-H. \& Do, T.-O. Direct synthesis and mechanism of the formation of mixed metal $\mathrm{Fe}_{2} \mathrm{Ni}-\mathrm{MIL}-88 \mathrm{~B}$. CrystEngComm 15, 9694-9703 (2013).

39. Zhu, X. et al. A superacid-catalyzed synthesis of porous membranes based on triazine frameworks for $\mathrm{CO}_{2}$ separation. J. Am. Chem. Soc. 134, 10478-10484 (2012).

40. Li, M.-X., Miao, Z.-X., Shao, M., Liang, S.-W. \& Zhu, S.-R. Metal-Organic frameworks constructed from 2,4,6-Tris(4-pyridyl)-1,3,5-triazine. Inorg. Chem. 47, 4481-4489 (2008).

41. Agenet, N. et al. Cotrimerizations of Acetylenic Compounds. in Organic Reactions 68, Ch 1: 1-302 (John Wiley \& Sons, Inc., 2004).

\section{Acknowledgements}

This research was supported by the '973 Project' (2014CB845602 and 2012CB821706) and NSFC $(21225105,21290173$, and 21473260).

\section{Author contributions}

J-.P.Z. conceived and designed the experiments. Y-.S.W., M.Z., and T-.Y.L. conducted synthetic experiments. Y-.S.W., M.Z., R-.B.L. and J-.P.Z. performed the physical measurements and crystal-structure analyses. P-.Q.L. performed sorption measurements Y-.S.W., G.S., J-.P.Z., and X-.M.C. co-wrote the manuscript. All authors discussed the results and commented on the manuscript.

\section{Additional information}

Accession codes. The X-ray crystallographic coordinates for structures reported in this study have been deposited at the Cambridge Crystallographic Data Centre (CCDC), under deposition numbers 1415803-1415810. These data can be obtained free of charge from The Cambridge Crystallographic Data Centre via www.ccdc.cam.ac.uk/ data_request/cif.

Supplementary Information accompanies this paper at http://www.nature.com/ naturecommunications

Competing financial interests: The authors declare no competing financial interests.

Reprints and permission information is available online at http://npg.nature.com/ reprintsandpermissions/

How to cite this article: Wei, Y.-S. et al. Coordination templated $[2+2+2]$ cyclotrimerization in a porous coordination framework. Nat. Commun. 6:8348 doi: $10.1038 /$ ncomms9348 (2015)

(c) (i) This work is licensed under a Creative Commons Attribution 4.0 International License. The images or other third party material in this article are included in the article's Creative Commons license, unless indicated otherwise in the credit line; if the material is not included under the Creative Commons license, users will need to obtain permission from the license holder to reproduce the material. To view a copy of this license, visit http://creativecommons.org/licenses/by/4.0/ 